\title{
Roles of $\gamma \delta$ T Cells in Cardiovascular Damage Induced by Lupus Erythematosus
}

\author{
Feng Chen ${ }^{1}$, Zhen Tian $^{2}$ \\ ${ }^{1}$ Dermatology Department, China-Japan Union Hospital of Jilin University, Changchun City, Jilin Province, \\ 130033, China \\ ${ }^{2}$ Cardiology Department, China-Japan Union Hospital of Jilin University, Changchun City, Jilin Province, \\ 130033, China
}

Keywords: $\gamma \delta \mathrm{T}$ cells; systemic lupus erythematosus; cardiovascular damage; clinical significance

\begin{abstract}
Objective: To investigate the role of $\gamma \delta \mathrm{T}$ cells in pathogenesis by checking the number and apoptosis rate of $\gamma \delta \mathrm{T}$ cells, $\mathrm{V} \delta 1+, \mathrm{V} \delta 2+$ cells in peripheral blood of patients with cardiovascular damage caused by systemic lupus erythematosus. Methods: 45 patients (23 active and 22 inactive) and 22 healthy persons (control group) were selected. The absolute count, percentage and apoptosis rate of $\gamma \delta \mathrm{T}$ cells in active, inactive and control peripheral blood were analyzed by flow cytometry. The absolute counts of $\mathrm{V} \delta 1+$ cells and $\mathrm{V} \delta 2+$ cells in peripheral blood of active, inactive and control groups were analyzed. Results: The absolute number of peripheral blood $\gamma \delta \mathrm{T}$ cells in active patients was significantly lower than that in inactive patients, and both of them were lower than those in control group. The apoptosis rate of $\gamma \delta \mathrm{T}$ cells in active phase was higher than that in inactive phase and control group. The number of $\mathrm{V} \delta 1+$ cells in active phase was lower than that in inactive phase and control group, and the number of $\mathrm{V} \delta 2+$ cells in active phase and inactive phase was lower than that in control group. The number of $\mathrm{V} \delta 1+$ cell in active phase was more than that in $\mathrm{V} \delta 2+$ cell. Conclusion: The number and proportion of $\gamma \delta \mathrm{T}$ cells in peripheral blood of patients with cardiovascular damage caused by systemic lupus erythematosus decreased, and the number of $\gamma \delta \mathrm{T}$ cells and $\mathrm{V} \delta 1+, \mathrm{V} \delta 2+$ subsets in peripheral blood of patients with systemic lupus erythematosus were abnormal. Apoptosis of $\gamma \delta \mathrm{T}$ cells in peripheral blood of patients with cardiovascular damage caused by systemic lupus erythematosus increased, resulting in overexpression of cells and disorder of immune system.
\end{abstract}

\section{Introduction}

Systemic lupus erythematosus is an autoimmune disease involving connective tissue of the whole body and the heart is its important target organ. In recent years, because of the prolongation of the survival period and the improvement of the breaking method, the mortality of cardiovascular diseases in systemic lupus erythematosus have increased, and it has become the third leading cause of death in systemic lupus erythematosus. This paper discusses the role of $\gamma \delta \mathrm{T}$ cells in pathogenesis by detecting the number and apoptosis rate of $\gamma \delta \mathrm{T}$ cells, $\mathrm{V} \delta 1+, \mathrm{V} \delta 2+$ cells in peripheral blood of patients with cardiovascular damage caused by systemic lupus erythematosus.

\section{Data and Methods}

\subsection{Clinical data.}

All specimens and health controls were collected from patients from October 2015 to December 2017 in the immune and Rheumatological Department of our hospital, as well as from normal medical examiners at the physical examination Center. Each group was divided into the following two groups: (1) Experimental group: 45 patients with cardiovascular damage caused by systemic lupus erythematosus, including 22 females and 23 males, with an average age of (31 \pm 4$)$ years. The diagnosis is in accordance with the revised classification criteria of the American Society of Rheumatology. No glucocorticoids or immunosuppressants were taken in the selected cases, and co-infection, neoplasms and other autoimmune diseases and chronic diseases were excluded. (2) 
Healthy control group: 22 normal subjects, 10 males and 12 females were selected. The mean age was (32 \pm 5$)$ years old, there was no family history of rheumatism, and there was no significant difference in age and sex. This study obtained the informed consent of all subjects.

\subsection{Method.}

Specimen collection: before the experiment, the fasting elbow venous blood was collected at 5 $\mathrm{ml}$ in the morning. The blood was placed in the $15 \mathrm{ml}$ centrifuge tube containing 0.3mlEDTA anticoagulant and mixed upside down. After specimen collection, the cells to be tested were stained with FITC-CD3,APC- $\gamma \delta$ and APC-CD3, FITC- binding protein V labeled with the corresponding mouse anti-human isothiocyanate photoluminescence, and then the suspension was made into a single cell suspension for computer detection. In flow cytometry, the sample is pressed into the flow chamber at a certain pressure, and the phosphoric acid buffer without cells is ejected from the sheath at high pressure. The inlet direction of the sheath liquid tube is at a certain angle to the flow of the sample to be tested. The sheath fluid can flow around the sample at high speed, and the cells to be tested are arranged in one line under the sheath fluid, and passed through the detection area in turn. The laser is used as the light source in the flow cytometry. The laser beam is assembled and shaped, and the cells stained by fluorescence produce scattered light and excited fluorescence. Both of these signals are accepted by both the forward transistor and the $90{ }^{\circ} \mathrm{C}$ photomultiplier tube. The light scattering signal basically reflects the size of the cell volume, and the direction of the light burst signal is perpendicular to the laser beam, and the burning signal is formed through the separation of the double color reflector and the bandpass filter. The intensity of the immolation signal represents the strength of the surface antigen of the cell membrane or the concentration of the substance in its nucleus, which can be converted into an electrical signal after being received by a photomultiplier tube, and then converted into a digital signal that can be recognized by a computer through an analog-to-digital converter. The computer can display the results of analysis by processing all kinds of signals measured by computer.

\subsection{Statistical treatment.}

The experimental data are analyzed by software, the measurement data are expressed by the standard deviation of mean soil, the rate or percentage of counting data is expressed, the comparison between groups is tested, and the analysis of variance is used to compare the mean of many groups. $\mathrm{P}<0.05$ indicated that the difference was statistically significant.

\section{Results}

Detection of absolute number of $\gamma \delta$ T cells by flow cytometry. The absolute number of $\gamma \delta \mathrm{T}$ cells in peripheral blood of patients with cardiovascular damage caused by active systemic lupus erythematosus $(1.8 \pm 0.2) \times 10^{7} / \mathrm{L}$ was significantly lower than that in inactive group $(5.2 \pm 0.9) \mathrm{x}$ $10^{7} / \mathrm{L}, \mathrm{P}<0.05$. Both of them were lower than those in the healthy control group $(14.5 \pm 5.2) \times 10^{7} / \mathrm{L}$ $(\mathrm{P}<0.05)$. The percentage of $\gamma \delta \mathrm{T}$ cells in peripheral blood of patients with cardiovascular damage caused by active systemic lupus erythematosus was $(0.6 \pm 0.2) \%$, which was lower than that in inactive phase $(1.2 \pm 0.3) \%, \mathrm{P}<0.05$, and healthy control group $(3.2 \pm 1.8) \%, \mathrm{P}<0.05$. There was no significant difference between inactive systemic lupus erythematosus patients and healthy controls.

\subsection{Detection of absolute numbers of $\mathrm{V} \delta 1+$ cells and $\mathrm{V} \delta 2+$ cells by flow cytometry.}

The count of $\mathrm{V} \delta 1+$ cells in active phase $(1.0 \pm 0.2) \times 10^{7} \% \mathrm{~L}$ was lower than that in healthy control group and inactive stage, $(4 \pm 3.1) \times 10^{7} \% \mathrm{~L},(2.0 \pm 0.5) \times 10^{7} \% \mathrm{~L}, \mathrm{P}<0.05$. The counts of $\mathrm{V} \delta 2+$ cells in active and inactive phases $(0.5 \pm 0.3) \times 10^{7} / \mathrm{L},(1.5 \pm 0.9) \times 10^{7} / \mathrm{L}$ were lower than those in healthy controls $(8.3 \pm 2.8) \times 10^{7} / \mathrm{L},(\mathrm{P}<0.05)$. In inactive patients, $\mathrm{V} \delta 1+$ cells $(2.0 \pm 0.5) \times 10^{7} / \mathrm{L}$ were more than $\mathrm{V} \delta 2+\mathrm{T}$ cells $(1.5 \pm 0.9) \times 10^{7} / \mathrm{L}$, but there was no statistical significance $(\mathrm{P}>0.05)$.

\subsection{Detection of apoptosis rate of $\gamma \delta \mathrm{T}$ cells by flow cytometry.}

The apoptosis rate of $\gamma \delta$ T cells was $(19.3 \pm 8.5) \%$ in active patients, $(11.2 \pm 1.5) \%$ in inactive 
patients and $(6.5 \pm 1.5) \%$ in healthy controls $(\mathrm{P}<0.05)$. There was no significant difference in the apoptosis rate of $\gamma \delta \mathrm{T}$ cells between inactive patients and healthy controls $(\mathrm{P}>0.05)$.

\section{Discusses}

Systemic lupus erythematosus is an autoimmune disease with multiple organ damage. As a special $\mathrm{T}$ cell subgroup, $\gamma \delta \mathrm{T}$ cells mainly recognize antigens by non-major histocompatibility complex, and it plays an important role in the occurrence of anti-infection immunity, anti-tumor immunity and autoimmune diseases. $\gamma \delta \mathrm{T}$ cells account for $0.5 \%$ of $\mathrm{T}$ cells in normal human peripheral blood, mainly distributed in skin, intestinal epithelium, lung, genital tract and splenic sinus. All $\gamma \delta \mathrm{T}$ cells had to undergo negative selection in thymus development to obtain tolerance to their own antigens, so they are CD4 double negative cells and only a few are CD8 cells. It has been confirmed that the positive selection of $\gamma \delta \mathrm{T}$ cells is opportunistic. Although its control mechanism is not clear, this may explain why many $\gamma \delta \mathrm{T}$ cells react with antigens without MHC restriction. In recent years, it has been found that the antigens recognized by $\gamma \delta \mathrm{T}$ cells have the following characteristics: (1) there are many kinds of recognition - peptide, non-protein antigen, non-peptide and so on; (2) the recognition of polypeptide antigen can be divided into two kinds - non MHC and MHC restriction. The recognition of polypeptide antigen is not restricted by MHC antigen. (3) the recognition of some changed autoantigens is of great significance in the pathogenesis of autoimmune diseases and tumors. $\gamma \delta \mathrm{T}$ cells recognize monolayers rather than peptide-MHC complexes. It is recognized that $\gamma \delta \mathrm{T}$ cell recognition is not restricted by $\mathrm{MHC}$, but it is bound to antigen surface or epitope in the same pattern of antibody.

$\gamma \delta \mathrm{T}$ cells are involved in the process of anti-tumor immunity, allergic autoimmune disease and other diseases. Its regulating effect is complicated - It can regulate immune response by membrane molecules and cytokines, also act as effector cells, and also plays a certain role in immune immunity and graft rejection. Some people think that if the cell is absent, the animal will face pathological immune incompetence, and its subset rerepair will reconstruct normal immune regulation. In some allergic diseases such as bronchial asthma, $\gamma \delta \mathrm{T}$ cells can inhibit the expansion of CD+4Th2A/BT cells and secrete cytokines such as IL-4, thus reducing the production of IgE by $\mathrm{B}$ cells. In addition, $\gamma \delta \mathrm{T}$ cells can also antagonize allergic reactions mediated by IgE and alleviate asthma attacks. Many early $\gamma \delta \mathrm{T}$ cell lines can dissolve tumor cells in a MHC independent manner, and $\gamma \delta \mathrm{T}$ cells are considered to have nonspecific immunosurveillance in anti-tumor immunity. Related researchers have found that $\gamma \delta \mathrm{T}$ cells play a key role in tumor immunity by the early sources of IFN-C, which in turn may regulate the tumor targeting function of ABT cells.

In recent years, more and more attention has been paid to the cardiac damage caused by systemic lupus erythematosus, but most of the heart damage is ignored because of the lack of clinical symptoms. The abnormal electrocardiogram mainly showed abnormal heart rate and ST-T, but could not reflect the cardiac involvement completely. As a noninvasive examination method, ultrasound can detect pericardial effusion, valvular involvement, pulmonary hypertension, myocardial involvement and abnormal cardiac function and it has became an important means of evaluating prognosis. In order to study the role of $\gamma \delta \mathrm{T}$ cells in cardiovascular damage induced by systemic lupus erythematosus, the results showed that the absolute number of $\gamma \delta \mathrm{T}$ cells in peripheral blood of active patients was significantly lower than that of inactive patients, and both of them were lower than the control group. The apoptosis rate of $\gamma \delta \mathrm{T}$ cells in active phase was higher than that in inactive phase and control group. The number of $V \delta 1+$ cells in active phase was lower than that in inactive phase and control group, and the number of $V \delta 2+$ cells in active phase and inactive phase was lower than that in control group. The number of $\mathrm{V} \delta 1+$ cell in active phase was more than that in $\mathrm{V} \delta 1+$ cell. The results showed that the number and proportion of $\gamma \delta \mathrm{T}$ cells in peripheral blood of patients with cardiovascular damage caused by systemic lupus erythematosus decreased, and the number of $\gamma \delta$ Tcells anV $\delta 1+, \mathrm{V} \delta 2+$ subsets in peripheral blood of patients with systemic lupus erythematosus were abnormal. Apoptosis of $\gamma \delta \mathrm{T}$ cells in peripheral blood of patients with cardiovascular damage caused by systemic lupus erythematosus increased, resulting in overexpression of cells and disorder of immune system. 


\section{References}

[1] Zeng Lihua, Yue Rui, long Xinxin, Peng Xuebiao. Expression of IL-17 T lymphocytes in peripheral blood of patients with systemic lupus erythematosus and its relationship with disease activity [J]. Guangdong Medicine, 2018 (07): 1008-1012.

[2] Cheng Jun, Zhang Wen, Cheng Qingtian, Yang Shumin. Expression of TGF- $\beta 1$ in $\gamma \delta$ T cells of mice with systemic lupus erythematosus [J]. Zhejiang Journal of Integrated Chinese and Western Medicine, 2012, 22(02): 89-91.

[3] Li Furong, Xu Hongxian. Effect of $\gamma \delta \mathrm{T}$ cells on activation and apoptosis of peripheral blood monocytes in patients with systemic lupus erythematosus [J]. Chinese Journal of Immunology, 2006 (11): 1048-1051.

[4] Peng Minmin, Chen Yan, Wang Renjie. Clinical significance of $\gamma \delta \mathrm{T}$ cell changes in peripheral blood of patients with systemic lupus erythematosus [J]. Journal of Armed Police Logistics College (Medical Edition), 2014, 23 (04): 284-286. 